\title{
Genetic versus environmental aetiology of the metabolic syndrome among male and female twins
}

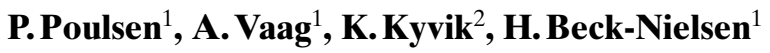 \\ ${ }^{1}$ Odense University Hospital, Department of Endocrinology, Odense, Denmark \\ ${ }^{2}$ Genetic Epidemiology Research Unit, Odense University, Odense, Denmark
}

\section{Abstract}

Aims/hypothesis. The aetiology of the metabolic syndrome including hyperinsulinaemia, glucose intolerance, dyslipidaemia, hypertension and obesity is not known. We studied the relative impact of genetic versus environmental factors for the development of the components in the syndrome among male and female twins.

Methods. A total of 303 elderly twin pairs participated in the study. We report concordances and heritability estimates of the components by classic twin analysis to assess the proportion of variation attributed to genetic factors.

Results. All components correlated significantly. The concordance rates for glucose intolerance, overall obesity and low HDL-cholesterol were significantly higher among monozygotic than dizygotic twins indicating a genetic influence on the development of these phenotypes. The heritability estimates for glucose concentration, BMI and HDL-cholesterol among monozygotic twins confirmed these findings.
The heritability estimates for waist-to-hip ratio, fasting insulin and triglycerides, however, were low, indicating a major environmental influence. We found a higher genetic influence on glucose intolerance and systolic blood pressure and a lower genetic influence on low HDL-cholesterol and diastolic blood pressure among male twins compared to female twins.

Conclusion/interpretation. Based on the correlations between the components in the syndrome, we propose a core complex including hyperinsulinaemia, obesity, hypertriglyceridaemia and low HDL-cholesterol with only weak associations to glucose concentrations and blood pressure levels. The study confirms the notion of a multifactorial aetiology of the components including genetic and non-genetic factors. The differences in aetiology between male and female twins indicate an influence of sex on several of the components in the metabolic syndrome. [Diabetologia (2001) 44: 537-543]

Keywords Twins, metabolic syndrome, heritability, aetiology.
Studies in several populations have shown clustering of hyperinsulinaemia, glucose intolerance, dyslipidaemia, hypertension, and obesity [1-6]. The clustering of these states of disease has been referred to as

Received: 2 October 2000 and in revised form: 2 January 2001

Corresponding author: Dr. P. Poulsen, Odense University Hospital, Department of Endocrinology, Sdr. Boulevard, Odense, Denmark

Abbreviations: MZ, Monozygotic; DZ, dizygotic; WHR, waistto-hip ratio; HOMA, homeostasis model assessment; HDL, high density lipoprotein. "the insulin resistance syndrome" or "the metabolic syndrome".

All of the individual components of the metabolic syndrome are considered to be risk factors for the development of cardiovascular disease. Currently, however, it is not known which of the components are primary and perhaps genetically determined and which are secondary. It has been proposed that insulin resistance itself, or its concomitant hyperinsulinaemia, could be the primary factor(s) or denominator(s) predisposing to the full blown syndrome in subjects exposed to the westernised lifestyle. To improve our knowledge about causality and interactive mecha- 
nisms among the various individual components of the metabolic syndrome, we used a classic twin study design to estimate the relative impact of genetic versus non-genetic factors for the development of the metabolic, haemodynamic and anthropometric abnormalities in the syndrome.

Of note, the twin sample was population-based using the currently updated Danish Twin Register to avoid ascertainment bias [7,8]. The aetiology of the various components was assessed separately among male and female twins in order to disclose a putative influence of sex on aetiology. In our study we report prevalences, concordances and heritability estimates of the various components by means of classic twin analysis.

\section{Subjects and methods}

Subjects. The twin sample was identified through the Danish Twin Register [7, 8] and included 3074 monozygotic (MZ) and dizygotic (DZ) twins (i.e. 1537 pairs) of similar sex born in Funen county, Denmark, between 1931 and 1940 or born in the whole of Denmark between 1921 and 1930 [9].

Each twin was asked in a mailed questionnaire whether they were willing to participate in a clinical examination involving a standard oral glucose tolerance test (OGTT), blood lipid profile, blood pressure and anthropometric measurements.

A total of 125 monozygotic (men: 62, women: 63) and 178 dizygotic (men: 86 , women: 92 ) twin pairs participated in the clinical examination. The mean age among the twin sample was 67.0 years (range 55-74 years). The subpopulation of twin pairs that were clinically examined did not differ in age or self-reported diabetes prevalence compared with the responding non-examined group of twins. Zygosity status was established by the similarity method where twins were asked about physical similarity and mistaken identity [10]. This method has been evaluated by comparison with serological zygosity testing, showing a misclassification rate below $5 \%$. The MZ:DZ ratio and male:female ratio was as expected.

The protocol was approved by the regional ethics committees and the study was conducted according to the principles of the Helsinki Declaration.

\section{Methods}

Weight and height were measured with the subject in lightweight clothes with the shoes removed and the body mass index (BMI) [weight $(\mathrm{kg}) /$ height $\left(\mathrm{m}^{2}\right)$ ] was calculated. Overall obesity was defined as a BMI greater than $27 \mathrm{~kg} / \mathrm{m}^{2}$ for men and a BMI greater than $25 \mathrm{~kg} / \mathrm{m} 2$ for women. Waist circumference was measured using a soft tape on standing subjects midway between the lowest rib and the iliac crest. Hip circumference was measured over the widest part of the gluteal region, and the waist-to-hip ratio (WHR) was calculated accordingly. Abdominal obesity was defined as a waist-to-hip ratio greater than 0.95 for men and greater than 0.85 for women. Blood pressure measurements were taken on the seated participant after a 30 min rest using a random-zero mercury sphygmomanometer (Hawksley, England). Three readings were recorded and the mean was used in the analysis. Hypertension was de- fined according to the World Health Organisation (WHO) criteria (i.e. systolic blood pressure $\geq 140 \mathrm{mmHg}$ and diastolic blood pressure $\geq 90 \mathrm{mmHg}$ and/or antihypertensive medication [11].

Subjects underwent a standard $75 \mathrm{~g}$ oral glucose tolerance test (OGTT) after a 10 to $12 \mathrm{~h}$ overnight fast. Peripheral venous blood was taken before oral glucose ingestion and 120 min later. Plasma glucose concentrations were analysed by the glucose dehydrogenase oxidation method. Glucose intolerance (impaired glucose intolerance or overt Type II (non-insulin-dependent) diabetes mellitus) was defined according to the current WHO criteria (i.e. $120 \mathrm{~min}$ post OGTT plasma glucose $\geq 7.8 \mathrm{mmol} / \mathrm{l}$ ) [12]. Plasma insulin concentrations were measured using a two-site, two-step, time resolved immunofluoremetric assay (DELFIA) as described previously $[9,13]$. Hyperinsulinaemia was defined as a fasting plasma insulin value of $55 \mathrm{pmol} / \mathrm{l}$ or more, equivalent to the upper $25 \%$ of the investigated cohort. We used fasting hyperinsulinaemia as an estimate for insulin resistance. Single fasting plasma insulin samples have been shown to correlate inversely with insulin sensitivity or whole-body glucose uptake measured using euglycaemic-hyperinsulinaemic clamp or frequently sampled intravenous glucose tolerance test methods specifically in insulin resistant subjects [14]. We applied the HOMA approach in assessing insulin sensitivity [15]. The results using this method did not differ from those obtained using the fasting insulin alone. As the HOMA approach has not been proven superior to fasting insulin (both being indirect measures) in assessing insulin sensitivity, the data are not included in the Results. The fasting blood samples were furthermore analysed for serum triglycerides and HDL-cholesterol using commercial kits from Boerhinger Mannheim, Mannheim, Germany. Dyslipidaemia was defined as plasma triglyceride concentrations of $1.7 \mathrm{mmol} / \mathrm{l}$ or more, and HDL-cholesterol of less than $1.1 \mathrm{mmol} / \mathrm{l}$ for women and of less than $0.9 \mathrm{mmol} / \mathrm{l}$ for men.

Statistical methods. Probandwise concordance i.e. the proportion of affected co-twins among co-twins of independently ascertained probands and $95 \%$ confidence intervals were estimated for $\mathrm{MZ}$ and $\mathrm{DZ}$ twin pairs and compared with a chisquared test. This estimates the risk of disease among co-twins of affected twins and is comparable to estimates of recurrence risk in other groups of relatives and in the general population.

Interclass correlation. Monozygotic twins have identical genotypes and any differences are theoretically due to environmental factors. Dizygotic twins, however, share on average $50 \%$ of their genes. The extent to which MZ twins are more alike than DZ twins is therefore presumed to reflect a genetic influence on the phenotype in question. Heritability $\left(h^{2}\right)$ expresses the proportion of the total variation of a trait attributable to genetic variation and can be estimated by comparing the similarity of a given phenotype within monozygotic and dizygotic twin pairs. Interclass correlations is a method to measure resemblance within twin pairs $[\mathrm{r}=\operatorname{cov}($ twin 1 , twin 2$) / \sqrt{ } \operatorname{var}($ twin 1$) \mathrm{x}$ var (twin 2)] [16]. Statistical comparisons of interclass correlations were made after transformation using the Fisher z-transformation. All tests applied were two-tailed and a $p$ value of less than 0.05 was considered statistically significant. The heritability is expressed as twice the difference of the interclass correlation of monozygotic and dizygotic twins $\left[\mathrm{h}^{2}=2\left(\mathrm{r}_{\mathrm{MZ}}-\mathrm{r}_{\mathrm{DZ}}\right)\right]$.

Before calculating interclass correlations and aetiological models the data for BMI, WHR, values of plasma glucose, plasma insulin, systolic blood pressure, diastolic blood pressure, triglycerides and HDL-cholesterol were applied logarithmic transformations to reduce skewness. The transformations yielded approximately normal distributions. 
Table 1. Phenotypic correlations for the components of the metabolic syndrome among 606 twins

\begin{tabular}{lccccccc}
\hline & F-insulin & 2 h-glucose & WHR & BMI & SBP & DBP & TG \\
\hline F-insulin & - & & & & & & \\
2 h-glucose & 0.32 & - & - & & & & \\
WHR & 0.30 & 0.23 & 0.35 & - & & & \\
BMI & 0.53 & 0.23 & 0.22 & 0.28 & - & & \\
SBP & 0.19 & 0.22 & 0.28 & 0.30 & 0.68 & - & \\
DBP & 0.20 & 0.11 & 0.22 & 0.36 & 0.20 & 0.28 & - \\
TG & 0.44 & 0.27 & -0.43 & -0.30 & -0.05 & -0.19 & -0.44 \\
HDL & -0.30 & -0.19 & & & & \\
\hline
\end{tabular}

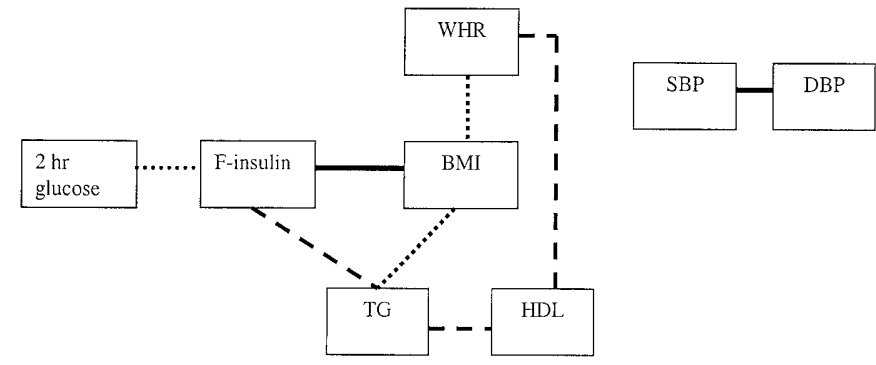

Fig.1. Graphic presentation of the highest Pearsons correlation coefficients explaining 10,15 and $20 \%$ of the variance $\geq 0.45-; \geq 0.39---; \geq 0.32 \ldots \ldots$

\section{Results}

Phenotypic correlations. The Pearson correlations of variables associated with the metabolic syndrome were similar among men and women and the results are therefore presented for the entire twin cohort. All components of the metabolic syndrome correlated statistically significantly with one another confirming the relation between these variables. Systolic blood pressure and HDL-cholesterol were the only pairs of variables that were not significantly correlated (Table 1).

The highest correlation coefficients, explaining an arbitrary defined level of at least 10,15 and $20 \%$ of the variance (i.e. $r \geq 0.32, r \geq 0.39$ and $r \geq 0.45$, respectively) are shown in Fig. 1. There were strong correlations between fasting insulin, WHR, BMI, triglycerides and HDL-cholesterol, which seem to be the core of the metabolic syndrome. Systolic and diastolic blood pressure are strongly correlated with each other, but only with the metabolic syndrome through a relatively weak correlation between diastolic blood pressure and BMI $(r=0.30$, not shown). Similarly, $2 \mathrm{~h}$ OGTT glucose concentrations were only related to this complex through the correlation with fasting insulin. No major correlation was seen between blood pressure levels and post OGTT glucose concentrations.

Prevalences. The prevalences of the components in the metabolic syndrome are shown in Table 2. One third of the cohort were glucose intolerant $(34.0 \%)$.
Table 2. Prevalence of the components included in the metabolic syndrome among all twins $(n=606)$ and separately among male $(n=296)$ and female $(n=310)$ twins

\begin{tabular}{llll}
\hline & \multicolumn{3}{l}{ Prevalence $(\%)$} \\
\cline { 2 - 4 } & All & Men & Women \\
\hline Hyperinsulinaemia & 25.0 & 24.7 & 25.5 \\
Glucose intolerance & 34.0 & 34.5 & 33.5 \\
Abdominal obesity & 30.7 & 38.5 & 23.2 \\
Overall obesity & 43.4 & 33.4 & 52.9 \\
Hypertension & 26.6 & 31.8 & 21.6 \\
Triglycerides $\uparrow$ & 23.9 & 23.3 & 24.5 \\
HDL cholesterol $\downarrow$ & 9.9 & 10.1 & 8.7 \\
\hline
\end{tabular}

Thirty-nine percent were obese whereas abdominal obesity was found in $30.7 \%$ of the cohort. Hyperinsulinaemia was found in $25.0 \%$ of the cohort according to the definition. Hypertension and hypertriglyceridaemia was found in $26.6 \%$ and $23.9 \%$ of the cohort, respectively. Low concentrations of HDL-cholesterol was observed among $9.9 \%$.

Concordances. The concordances for hyperinsulinaemia, abdominal obesity, hypertension and hypertriglyceridaemia concentrations were not significantly different among MZ and DZ twin pairs (Table 2). Furthermore the concordance rates for these components were similar among male and female twin pairs. The concordance rate for glucose intolerance, however, was significantly higher among MZ pairs than in DZ pairs (MZ 0.64 vs DZ $0.43, p<0.05$ ). When comparing separately among men and women we only found this difference among male twin pairs (MZ 0.70 vs DZ $0.41, p<0.05$ ), while the concordance rate for glucose intolerance was similar among female MZ and DZ twin pairs. The concordance rate for overall obesity was significantly higher among MZ compared to DZ pairs (MZ 0.71 vs DZ $0.56, p<0.05)$ indicating a genetic influence on the development of overall obesity. The difference between $\mathrm{MZ}$ and $\mathrm{DZ}$ twins was not apparent when analysing men and women, respectively. The concordance rate for low HDL-cholesterol concentrations was significantly higher among $\mathrm{MZ}$ compared to DZ twin pairs (MZ 0.55 vs DZ $0.18, p<0.05$ ). This difference was only consistent among female pairs when 
Table 3. Concordances of the components included in the metabolic syndrome among male $(n=148)$ and female $(n=155)$ twin pairs

\begin{tabular}{|c|c|c|c|c|c|c|}
\hline & \multicolumn{6}{|c|}{ Concordance rate } \\
\hline & \multicolumn{2}{|l|}{ All } & \multicolumn{2}{|l|}{ Men } & \multicolumn{2}{|c|}{ Women } \\
\hline & $\mathrm{MZ}$ & $\mathrm{DZ}$ & $\mathrm{MZ}$ & $\mathrm{DZ}$ & $\mathrm{MZ}$ & $\mathrm{DZ}$ \\
\hline Glucose intolerance & $0.64^{\mathrm{a}}$ & 0.43 & $0.70 *$ & 0.41 & 0.57 & 0.45 \\
\hline Abdominal obesity & 0.50 & 0.51 & 0.54 & 0.58 & 0.44 & 0.40 \\
\hline Overall obesity & $0.71^{\mathrm{a}}$ & 0.56 & 0.58 & 0.37 & 0.80 & 0.66 \\
\hline HDL cholesterol $\downarrow$ & $0.55^{\mathrm{a}}$ & 0.18 & 0.29 & 0.25 & $0.67^{\mathrm{a}}$ & 0.13 \\
\hline
\end{tabular}

${ }^{\mathrm{a}} p<0.05 \mathrm{MZ}$ vs DZ

Table 4. Interclass correlations and heritability estimates for continuous anthropometric and metabolic variables associated with the metabolic syndrome in MZ $(n=125)$ and DZ $(n=178)$ twin pairs

\begin{tabular}{lllll}
\hline & \multicolumn{2}{l}{ Interclass correlation } & & Heritability h2 \\
\cline { 2 - 3 } & MZ & DZ & & \\
\hline Body mass index (kg/m2) & 0.68 & 0.28 & 0.80 & $<0.001$ \\
Waist-to-hip ratio & 0.75 & 0.72 & 0.06 & $\mathrm{NS}$ \\
120 min post OGTT plasma glucose (mmol/l) & 0.52 & 0.26 & 0.52 & $\mathrm{~N}$ \\
Fasting plasma insulin (pmol/l) & 0.51 & 0.38 & 0.26 & $<0.001$ \\
Systolic blood pressure (mmHg) & 0.55 & 0.17 & 0.76 & $<0.01$ \\
Diastolic blood pressure (mmHg) & 0.40 & 0.07 & 0.66 & $\mathrm{NS}$ \\
Fasting plasma triglycerides (mmol/l) & 0.44 & 0.24 & 0.40 & $<0.001$ \\
Fasting plasma HDL cholesterol & 0.61 & 0.26 & 0.70 & \\
\hline
\end{tabular}

Table 5. Heritability estimates for continuous anthropometric and metabolic variables associated with the metabolic syndrome among male $(n=148)$ and female $(n=155)$ twin pairs separately

\begin{tabular}{lll}
\hline & Heritability h2 & Women \\
\cline { 3 - 3 } & Men & $0.90(0.59-1.21)$ \\
\hline Body mass index $(\mathrm{kg} / \mathrm{m} 2)$ & $0.58(0.40-0.76)$ & $0.10(0.07-0.13)$ \\
Waist-to-hip ratio & $0.22(0.09-0.34)$ & $0.46(0.32-0.60)$ \\
120 min post OGTT plasma glucose (mmol/l) & $0.62(0.45-0.79)$ & $0.14(0.01-0.27)$ \\
Fasting plasma insulin (pmol/l) & $0.36(0.16-0.56)$ & $0.58(0.42-0.74)^{\mathrm{a}}$ \\
Systolic blood pressure (mmHg) & $1.00(0.78-1.22)$ & $0.82(0.60-1.04)^{\mathrm{a}}$ \\
Diastolic blood pressure (mmHg) & $0.04(-0.26-0.34)$ & $0.34(0.21-0.47)$ \\
Fasting plasma triglycerides $(\mathrm{mmol} / \mathrm{l})$ & $0.50(0.29-0.71)$ & $0.84(0.60-1.09)$ \\
Fasting plasma HDL cholesterol (mmol/l) & $0.56(0.37-0.74)$ & \\
\hline
\end{tabular}

${ }^{\mathrm{a}} p<0.05$

analysing the two sex groups separately (MZ 0.67 vs DZ $0.13, p<0.05)$, whereas no difference was detected between male $\mathrm{MZ}$ and $\mathrm{DZ}$ pairs.

Heritability. The interclass correlations and heritability estimates shown in Table 4 are reported for continuos data and are therefore an estimate of the proportion of the total variation attributable to genetic variation for the phenotype in itself and not for a defined level of the phenotype in question. These results can therefore support the concordances but are not directly comparable as concordances are based on categorical data alone. The significant differences in interclass correlation between $\mathrm{MZ}$ and $\mathrm{DZ}$ pairs for $120 \mathrm{~min}$ post OGTT plasma glucose (MZ 0.52, DZ $0.26, p<0.05$ ) and BMI (MZ 0.68, DZ 0.28, $p<0.001)$ and the similar non-significant interclass correlations for WHR and fasting plasma insulin concentrations among MZ and DZ pairs were reported previously [21]. The heritability estimates for systolic (0.76) and diastolic (0.66) blood pressure and HDLcholesterol (0.70) were high reflecting the statistically significant difference in interclass correlation between MZ and DZ twins. The interclass correlation for plasma triglycerides was similar among $M Z$ and DZ twins indicating a major environmental aetiological factor. We subsequently calculated interclass correlations and heritability estimates separately for 
men and women. These analyses showed a significantly higher heritability for diastolic blood pressure among women compared to men (men 0.04 vs women 0.82 ), whereas systolic blood pressure had a higher heritability among men (men 1.00 vs women 0.58 ). These findings indicate an influence of sex on blood pressure levels.

\section{Discussion}

Previous epidemiological and metabolic studies have indicated a role for both genetic and environmental factors for the development of hyperinsulinaemia [9, 17-21], glucose intolerance [22-24], obesity [25-26], blood pressure [27-28] and dyslipidaemia [29]. The advantage of this twin study is that the ascertainment of twins (male and female) was done independent of disease status and all participants underwent a clinical examination including measures of all components included in the syndrome.

The significantly higher concordance rates for glucose intolerance, BMI and HDL cholesterol among MZ twins indicate a genetic influence on the development of these major components of the metabolic syndrome. Interestingly, the difference between MZ and DZ concordance rates for glucose intolerance was explained primarily by the concordance rate among male twins, whereas we found no evidence for the influence of genes on glucose tolerance in females. In contrast, the difference in concordance rates for HDL-cholesterol was only detected among female twins. The high heritabilities for HDL-cholesterol concentrations, indicating a major genetic component, are similar to results from previous twin studies. Previous studies have reported a heritability of $71 \%$ among younger female twins [30] and $76 \%$ and $55 \%$ among a younger and older twin group, respectively [29]. The concordance rate for BMI was not significantly higher among MZ twins when analysed among male and female twins independently, presumably due to the smaller number of twins in these comparisons.

No differences in concordances for hyperinsulinaemia, abdominal obesity, hypertension and triglycerides were found in this study indicating a quantitatively major environmental influence on those particular components of the metabolic syndrome. The interclass correlations and heritability estimates for the continuos levels of fasting plasma insulin, waistto-hip ratio and triglycerides confirmed this finding indicating that the continuous level as well as the phenotype defined by a threshold is primarily determined by environmental factors. Previously a heritability of $47 \%$ for fasting insulin concentration [21] and a heritability of $39 \%$ for insulin resistance estimated by homeostasis model assessment (HOMA) has been shown [20]. The major environmental con- tribution to the variation of plasma triglycerides among elderly twins also agrees with previous studies $[20,29]$. Low birth weight, physical inactivity and high fat diet are environmental factors potentially responsible for the development of hyperinsulinaemia, systolic blood pressure, abdominal obesity and hypertriglyceridaemia.

The differences in concordance rates or heritability estimates, or both, for glucose intolerance, low HDL-cholesterol concentration and systolic and diastolic blood pressure indicate that different aetiological (genetic and/or environmental) mechanisms are operating in men and women. A gender difference in the magnitude of genetic contribution to the variation of blood pressure levels has been proposed. Higher heritability estimates among women for both systolic and diastolic blood pressure have been reported [31]. Furthermore a previous study reported a higher heritability for systolic blood pressure and a lower heritability for diastolic blood pressure among men compared with women [20]. These results were not significant but are indeed in accordance with our findings. No previous study has to our knowledge reported differences in the aetiology of glucose intolerance and HDL-cholesterol concentrations among men and women. A sex hormonal influence on glucose and fat metabolism could explain the observed differences. The women participating in this study were postmenopausal (55-74 years). Therefore, no current oestrogen influence can explain the difference in mechanism. The amount and distribution of fat is, however, under sex hormonal influence and might influence glucose and fat metabolism directly or by influencing the expression of the responsible genes, explaining the sex differences. Testosterone has a well-described influence on fat distribution (i.e. higher waist-to-hip ratio) and the development of insulin resistance. Hyperandrogenicity in female rodents and humans is associated with insulin resistance and centralisation of body fat. Recently, a Swedish study showed that early testosterone imprinting of neonatal female rats is followed by insulin resistance and changes in body fat distribution in adult life [32]. Furthermore, sex specific heritability might also occur as a result of genetic imprinting [33], as mediated through methylations of DNA in specific genes on their coding regions, or perhaps in particular in their promotor regions regulating the expression of the genes.

The phenotypic correlations illustrate a core complex of abnormalities including insulin resistance, dyslipidaemia and obesity (i. e. fasting insulin, triglycerides, HDL-cholesterol, BMI and WHR) associated with relatively strong correlations. Glucose tolerance and blood pressure, however, are independently and peripherally associated to this complex within comparison rather weaker correlations. The Framingham Offspring Study has reported very similar associa- 
tions consistent with three distinct physiological domains i.e. a central metabolic syndrome, glucose intolerance and hypertension [6]. The findings could question the role of hypertension and glucose intolerance as core components in the metabolic syndrome. The results do not explain the causal connections between the variables or components, or both. However, one can hypothesise that subjects exposed to westernised lifestyle specifically develop abdominal obesity. Abdominal obesity could lead to hyperinsulinaemia and dyslipidaemia resulting in a core of abnormalities including obesity, dyslipidaemia and hyperinsulinaemia. In subjects genetically predisposed to hypertension, increased blood pressure could subsequently be a part of the syndrome. Type II diabetes could develop with time in subjects predisposed to beta-cell insufficiency or peripheral insulin resistance, or both [34].

We have recently reported some evidence for higher post glucose load plasma glucose and insulin concentrations among monozygotic twins compared to dizygotic twins indicating a higher degree of insulin resistance among monozygotic twins [35]. These findings imply a role of the intrauterine environment in the development of at least some of the components included in the metabolic syndrome. To which extent these findings question the validity of the classic twin approach used in our study is currently unknown.

Our study sample could be "survivors" and consequently differ from the deceased twins not participating in the study. Unfortunately, no data were available on the deceased twins regarding prevalence of the components included in the syndrome. An ongoing prospective study of the present twin sample will allow us to disclose an influence of age on the aetiology of the components included in the syndrome. Although we clinically examined a considerable number of twins, the number of affected twins of certain components was relatively small. Consequently, the statistical power for some comparisons might not have been optimal, and therefore smaller differences in concordance rates for example might have reached statistical significance if more twins had been included. Therefore we are well aware of the fact that a major genetic influence, for example, on a given phenotype does not rule out a concomitant environmental influence and vice versa. The classic twin study design, however, does provide an estimate of the magnitude of the genetic and/or environmental aetiological factors among the examined population.

In conclusion, the phenotypic correlations between the various components in the metabolic syndrome indicate a core complex including hyperinsulinaemia, obesity, hypertriglyceridaemia and low HDL-cholesterol concentrations with only weak associations to glucose concentrations and blood pressure levels. The study confirms the notion of a multi- factorial aetiology of the metabolic syndrome and its components including both genetic and non-genetic factors. The genetic effect on the development of several components is presumably heterogeneous. The number of genes involved (single and/or multiple genes) and the genetic interactions cannot be determined by our study. Furthermore, the differences in heritability between male and female twins propose an influence of sex on several of the components of the metabolic syndrome indicating an important role for sex-specific imprinting of metabolic pathways or genes controlling the metabolic syndrome.

Acknowledgements. The authors would like to acknowledge the expert technical assistance of L. Hansen, H. Hansen, C. B. Olsen and K. Dyregaard. This study was supported by grants from NOVO Foundation, Clinical Research Institute, Odense University and The Danish Diabetes Association and Sehested-Hansens Foundation.

\section{References}

1. Modan M, Halkin H, Almog S et al. (1985) J Clin Invest 75: 809-817

2. Reaven GM (1988) Role of insulin resistance in human disease. Diabetes 37: 1595-1607

3. Ferrannini E, Buzzigoli G, Bonadonna R et al. (1987) Insulin resistance in essential hypertension. N Engl J Med 317: 350-357

4. Ferrannini E, Haffner SM, Mitchell BD, Stern MP (1991) Hyperinsulinemia: the key feature of a cardiovascular and metabolic syndrome. Diabetologia 34: 416-422

5. DeFronzo RA, Ferrannini E (1991) Insulin resistance. A multifaceted syndrome responsible for NIDDM, obesity, hypertension, dyslipidemia and atherosclerotic cardiovascular disease. Diabetes Care 14: 173-194

6. Meigs JB, D'Agostino RB, Wilson PWF, Cupples LA, Nathan DM, Singer DE (1997) Risk Variable clustering in the insulin resistance syndrome. Diabetes 46: 1594-1600

7. Hauge M (1981) The Danish Twin Register. In: Mednick SA, Baert AE, Bachmann BP (eds) Prospective longitudinal research. An empirical basis for the primary prevention of psychosocial disorders. Oxford University Press, Oxford pp 217-221

8. Kyvik KO, Green A, Beck-Nielsen H (1995) The new Danish Twin Register: Establishment and analysis of twinning rates. Int J Epidemiol 24: 589-596

9. Poulsen P, Kyvik KO, Vaag A, Beck-Nielsen (1999) Heritability of non-insulin-dependent diabetes mellitus (NIDDM) and abnormal glucose tolerance - A populationbased twin study. Diabetologia 42: 139-145

10. Magnus P, Berg K, Nance WE (1983) Predicting zygosity in Norwegian twin pairs born 1915-1960. Clin Genet 24: 103-112

11. Chalmers J, MacMahon S, Mancia G et al. WHO-ISH Hypertension Guidelines Commitee (1999) World Health Organization - International Society of Hypertension guidelines for the management of hypertension. Clin Exp Hypertension 21: 1009-1060

12. Keen H (1992) Diabetes diagnosis. In: Alberti, KGMM, Defronzo RA, Keen H, Zimmet P (eds) International Textbook of Diabetes Mellitus. John Wiley \& son. Chichester pp 19-30 
13. Hemmilä I, Dakubu S, Mukkala V-M, Siitari H, Lövgren T (1984) Europium as a label in time-resolved immunoflourometric assays. Anal Biochem 137: 335-343

14. Laakso M (1993) How good a marker is insulin level for insulin resistance? Am J Epidemiol 137: 959-965

15. Matthews DR, Hosker JP, Rudenski AS, Naylor BA, Treacher DF, Turner RC (1985) Homeostasis model assessment: insulin resistance and beta-cell function from fasting plasma glucose and insulin concentrations in man. Diabetologia 28: 412-419

16. Neale MC, Cardon LR (1992) Data summary. In: Methodology for genetic studies of twins and families. Kluwer Academic Publishers, Dordrecht, Boston, London. NATO ASI series, pp 35-53

17. Laws A, Stefanick ML, Reaven GM (1989) Insulin resistance and hypertriglyceridemia in nondiabetic relatives of patients with non-insulin-dependent diabetes mellitus. J Clin Endocrinol Metab 69: 343-347

18. Haffner SM, Stern MP, Hazuda HP, Mitchell BD, Patterson JK (1988) Increased insulin concentrations in nondiabetic offspring of diabetic parents. N Engl J Med 319: 1297-1301

19. Vaag A, Henriksen JE, Madsbad S, Holm N, Beck-Nielsen $\mathrm{H}$ (1995) Insulin secretion, insulin action and hepatic glucose production in identical twins discordant for NIDDM. J Clin Invest 95: 690-698

20. Hong Y, Pedersen NL, Brismar K, de Faire U (1997) Genetic and environmental architecture of the features of the insulin-resistance syndrome. Am J Hum Genet 69: 143-152

21. Mayer EJ, Newman B, Austin MA et al. (1996) Genetic and environmental influences on insulin levels and the insulin resistance syndrome: an analysis of women twins. Am J Epidemiol 143: 323-332

22. Edwards KL, Austin MA, Newman B, Mayer E, Krauss RM, Selby JV (1994) Multivariate analysis of the insulin resistance syndrome in women. Arterioscler Thromb 14: 1940-1945

23. Newman B, Selby JV, King MC, Slemenda C, Fabsitz R, Friedman GD (1987) Concordance for Type II (non-insulin-dependent) diabetes mellitus in male twins. Diabetologia 30: 763-768
24. Kaprio J, Tuomilehto J, Koskenvuo M et al. (1992) Concordance for Type I (insulin-dependent) and Type II (non-insulin-dependent) diabetes mellitus in a population-based cohort of twins in Finland. Diabetologia 35: 1060-1067

25. Bouchard C, Tremblay A, Després J-P et al. (1990) The response to long-term overfeeding in identical twins. N Engl J Med 322: 1477-1482

26. Selby JV, Newman B, Quesenberry CP et al. (1990) Genetic and behavioral influences on body fat distribution. Int $\mathrm{J}$ Obes 14: 593-602

27. Fagard R, Brguljan J, Staessen J et al. (1995) Heritabilty of conventional and ambulatory blood pressures. Hypertension 26: 919-924

28. Hong Y, de Faire U, Heller DA, McClearn GE, Pedersen N (1994) Genetic and environmental influences on blood pressure in elderly twins. Hypertension 24: 663-670

29. Heller DA, de Faire U, Pedersen NL, Dahlen G, McClearn GE (1993) Genetic and environmental influences on serum lipid levels in twins. N Engl J Med 328: 1150-1156

30. Williams PD, Puddey IB, Martin NG, Beilin LJ (1993) Genetic and environmental covariance of serum cholesterol and blood pressure in female twins. Atherosclerosis 100: 19-31

31. McIlhany ML, Shaffer JW, Hines EA (1975) The heritability of blood pressure: an investigation of 200 pairs of twins using the cold pressor test. Johns Hopkins Med J 136: 57-64

32. Nilsson C, Niklasson M, Eriksson E, Björntorp P, Holmäng A (1998) Imprinting of female offspring with testosterone results in insulin resistance and changes in body fat distribution at adult age in rats. J Clin Invest 101: 74-78

33. Fenger M, Vaag A (1999) Epigenetics: DNA methylation. Ugeskr Laeger 161: 3823-3827

34. Beck-Nielsen H, Groop L (1994) Metabolic and genetic characterization of prediabetic states. Sequence of events leading to non-insulin-dependent diabetes mellitus. J Clin Invest 94: 1714-1721

35. Poulsen P, Vaag A, Beck-Nielsen (1999) Does zygosity influence the metabolic profile in twins. BMJ 319: 151-154 